\title{
Perfusion C evaluation in experimentally induced testicular torsion: proceed with caution
}

\author{
Walid A. Farhat, MD, FRCSC
}

See related article on page 383

As imaging evolves and an accurate preoperative diagnosis is made, less surgery will need to be performed. However, in the testicular torsion scenario the stakes are high, and there is the need for a $100 \%$ sensitive test. Ovali and colleagues ${ }^{1}$ use advanced technology to discriminate testicular torsion from non-torsion. The animal model used is a valid animal model, but there are many variables to take into account. Although an advanced diagnostic modality may be useful in indeterminate cases of testicular torsion, there are some issues that need to be resolved prior to incorporating this modality into daily practice.

Firstly, in clinical practice, we have yet to define what the indeterminate cases with testicular torsion are: Are these patients experiencing minimal pain with findings suggestive of torsion but not clinically confirmed torsion, or are they patients presenting to the emergency room with vague scrotal complaints and discomfort? Secondly, prior to adopting a new technology, we need to be vigorous in understanding its utility in comparison to the available diagnostic modalities and surgical findings. Ultrasound (US), Doppler and nuclear scans are not yet routine tests done in most tertiary care centres. They are ancillary tests that may help rule out torsion, but they are not found to be clinically useful in emergency room settings. The authors provide an elaborate list of different measurements when performing computed tomography (CT) scans in this experimental study, but does this list entail higher radiation doses and possibly longer times in the radiology suite (time is a known factor to be detrimental to testicles in children with presumptive diagnosis of torsion)? Since the introduction of ALARA (As Low as Reasonably Achievable), in 2001 by the Society for
Pediatric Radiology, physicians and radiologists have been collaborating to lower radiation exposure by avoiding unnecessary CT examinations. Radiologists at our institution actively screen $\mathrm{CT}$ requests, stating that the risks outweigh the benefits for CT scans compared to other radiologic examinations (US, magnetic resonance imaging and others). Furthermore, children are known to be 10 times more sensitive to radiation than middle-aged adults; therefore. the authors need to be cognizant about the dose of the CT scan in this setting and the duration of the test. Finally, and most importantly, in an era of economic crisis, the cost-effectiveness of an advanced technology needs to be thoroughly investigated. The authors touched briefly on these two issues, but more accurate data needs to be investigated in future studies should CT become a diagnostic tool for testicular torsion in children.

From the Department of Urology, The Hospital for Sick Children (SickKids), Toronto, ON

This paper has been peer-reviewed.

Competing interests: None declared.

\section{Reference}

1. Ovali GY, Yilmaz 0, Tarhan S, et al. Perfusion CT evaluation in experimentally induced testicular torsion. Can Urol Assoc J 2009;3:383-6.

Correspondence: Dr. Walid Farhat, Department of Urology, The Hospital for Sick Children, Main Floor Black Wing Room M299-555, University Avenue, Toronto, ON M5G 1X8; fax: 416-8136461; walid.farhat@sickkids.ca 